\title{
MicroRNA-150 enhances radiosensitivity by inhibiting the AKT pathway in NK/T cell lymphoma
}

\author{
Shao Jie Wu' ${ }^{1 *}$ Jun Chen², BingYi Wu' ${ }^{1}$ Yu Jue Wang ${ }^{3}$ and Kun Yuan Guo
}

\begin{abstract}
Background: Radioresistance is a major challenge during the treatment of NKTT cell lymphoma. This study aimed to investigate the potential role of MicroRNA-150 (miR-150) in increase the sensitivities of NK/T cell lymphoma to ionizing radiation.

Results: In this study, we found that miR-150 was significantly decreased in NK/T cell lymphoma tissues and cell lines. Low expression of miR-150 was positively associated with therapeutic resistance in 36 NK/T cell lymphoma cases. Our further in vitro and in vivo studies illustrated that overexpression of miR-150 substantially enhanced the sensitivity of NK/T cell lymphoma cells to ionizing radiation treatment. Furthermore, luciferase reporter assays in NK/ T cell lymphoma cells transfected with the AKT2 or AKT3 three prime untranslated region reporter constructs established AKT2 and AKT3 as direct targets of miR-150. The phosphatidylinositol 3-kinase inhibitor LY294002 was used to inhibit Akt to verify miR-150 increase NKJT cell lymphoma cell radiorsensitivity through suppress the PI3K AKT/mTOR pathway.
\end{abstract}

Conclusions: Taken together, this study demonstrates that miR-150 might serve as a potential therapeutic sensitizer through inhibition of the AKT pathway in NK/T cell lymphoma treatment.

Keywords: MicroRNA-150(miR-150), NK/T cell lymphoma, PI3K/AKT/mTOR pathway, Radiosensitivity, Apoptosis

\section{Background}

NK/T cell lymphoma (NKTL) is diagnosed more commonly in Asia and Latin America than in Western countries and accounts for $5-10 \%$ of all malignant lymphomas in China $[1,2]$. NKTL is a highly invasive tumor with a short doubling time and poor prognosis. Radiotherapy is usually regarded as the main treatment, however, the cellular response to irradiation is complex. The treatment effects also depend on many factors. For example, the dose, dose rate, and fractionation play an equally important role in deciding the fate of the cell. One of the main causes of failure in radiotherapy is radioresistance [3-5]. Therefore, to develop effective radiotherapy strategies in the future, the knowledge of how radioresistance develops at the molecular level is needed.

\footnotetext{
*Correspondence: wshj510515@163.com

'Department of Hematology, Southern Medical University, Zhujiang Hospital, 253\# industry road, Guangzhou, Guangdong 510282, China

Full list of author information is available at the end of the article
}

MicroRNAs (miRNAs) are a class of short, highly conserved, noncoding RNAs that function as negative post transcriptional regulators of the target genes [6]. Recently, growing evidence has shown that miRNAs are aberrantly expressed in the development and progression of variety of human cancers [7]. Increasing evidence has confirmed that miRNAs can modulate the radiosensitivity of cancer cells, suggesting the potential to improve the efficacy of radiotherapy $[8,9]$. miR- 150 has recently been identified as a key regulator of immune cell differentiation and activation, as it is preferentially expressed in mature B and T cells as well as NK cells and other cell types of the hematopoietic system [10-12]. MicroRNA-150 (miR-150) has been validated as an oncogene or tumor suppressor in several human malignancies [13, 14]. Previous reports have documented that miR-150 was frequently downregulated in NKTL [15]. However, the role and mechanisms of miR150 in NKTL therapeutic effects remain elusive. This study aimed to investigate the potential role of miR-150 in 
increasing the sensitivities to ionizing radiation and through suppress the PI3K/AKT/mTOR pathway of NKTL.

\section{Methods}

\section{Cell lines and cell cultures}

We used 5 cell lines as NK/T-cell lymphoma leukemia cell lines, which are commonly showing $\mathrm{CD}^{+}, \mathrm{sCD}^{-}, \mathrm{CD} \varepsilon^{+}$, $\mathrm{CD} 5^{-}, \mathrm{CD}_{5} 6^{+}, \mathrm{TCR} \alpha \beta^{-}$and $\mathrm{TCR} \gamma \delta^{-}$phenotypes, including KHYG-1, NK-92, HANK-1, SNK-1, SNK-6. Of these, HANK-1, SNK-1 and SNK-6 are EBV ${ }^{+}$. Those cell lines were maintained in alpha-MEM (Minimum Essential Medium) supplemented with $10 \%$ fetal bovine serum (Invitrogen, Carlsbad, CA) and $100 \mathrm{U} / \mathrm{ml}$ recombinant IL-2. NK cells showing the $\mathrm{sCD}^{-}$and $\mathrm{CD}^{-} 6^{+}$phenotype were collected from 6 healthy donors as a control using a magnetic cell sorting system and flow cytometry (Additional file 1: Figure S1). All cell lines were cultured at $37^{\circ} \mathrm{C}$ in a humidified atmosphere of $5 \% \mathrm{CO}_{2}$.

\section{Patients and therapy response evaluation}

Paraffin-embedded tissues of 36 primary NKTL were collected from 22 nasal, 6 pharyngeal, 5 gastrointestinal tract and 3 lung, and obtained from the Department of Pathology of Zhujiang Hospital, Southern Medical University, between January 2010 and January 2016. These were assessed according to Ann Arbor Staging [16]. None of the patients received radiotherapy before biopsy sampling. The response of radiotherapy was assessed clinically for primary lesion based on CT (computed tomography) and PET (positron emission tomography)/CT 1 month after treatment according to the following criteria: complete response (CR) was defined as the complete disappearance of all assessable lesions, partial response (PR) was defined as a reduction of the sum of the lesions by $50 \%$ or more and no progression of assessable lesions, stable disease (SD) was indicated by a reduction of $<50 \%$ or an increase of $<25 \%$ in tumor size. All these conditions were required to last for at least 4 weeks with no appearance of new lesions. Progressive disease (PD) was defined as an increase of $25 \%$ in tumor size or the appearance of new lesions. The PR, SD and PD define as Non-CR.

\section{Quantificational real-time polymerase chain reaction (qRT-PCR) for quantitative analysis of miR-150}

Total RNA was extracted from the cells and tissues using TRIzol reagent according to the manufacturer's protocol. The reverse transcription and polymerase chain reaction (PCR) primers for miR-150 and U6 were obtained from RiBoBio. Reverse transcription primersfor miR-150 were 5' -CTCAACTGGTGTCGTGGAGTCGGCAATTCAGT TGAGCACTGGTA-3'. The PCR primers for miR-150 were 5'-ACACTCCAGCTGGGTCTCCCAACCCTTGT ACCA-3' and 5'-CTCAACTGGTGTCGTGGA-3'. The PCR primers for U6 were $5^{\prime}$-CTCGCTTCGGCAG
CACA-3' and 5' ${ }^{\prime}$-AACGCTTCACGAATTTGCGT-3'. The cDNA library was synthesized using the PrimeScript RT reagent kit. For quantification of mature miRNA, cDNA was generated using specific stem-loop universal primers. qRT-PCR for miRNA and mRNA was performed using SYBR Premix Ex Taq II and was measured using an ABI 7500 Sequence Detection System. U6 was used as the internal control.

\section{Plasmid construction, lentivirus production and transduction}

The hsa-miR-150-5p gene fragment was synthesized chemically and cloned into lentiviral vector pLVXZsGreen-miRNA-Puro. The constructed recombinant plasmid pLVX-ZsGreen-miR-150-5p-Puro/pLVX-ZsGre en-miRNA-Puro, pspax2 and pMD2G was transfected to packaging cell line $293 \mathrm{~T}$, and the packaged virus was determined for titer. Recombinant lentivirus particles harvested and concentrated, then $1 \times 10^{4} / \mathrm{L}$ NK-92/ Hank-1 cells were seeded into 24 well plates, we added the virus suspension into plates by Polybrene concentration gradient dilution method, the infection rate was low (about 30-40\%) after $72 \mathrm{~h}$ by fluorescence microscope, then GFP-positive cells were selected transfer to 96 well plates for further culture by flow cytometry cell sorting, GFP-positive clones were expanded to construct stable cell line, and tested for expression level of miR-150 by qRT-PCR. The experiment was divided into three groups, including mock group, miR-control group, and miR-150 group.

\section{Cell proliferation assay}

$1 \times 10^{6}$ NK-92 or Hank-1cells of above three groups were irradiated for each well with $0,2,4,6,8$, and $10 \mathrm{~Gy}$, respectively. Cells were subjected to radiation exposure by a $6-\mathrm{Mv} \mathrm{X}$ ray (isocentric) linear accelerator. The dose rate was fixed as $300 \mathrm{cGy} / \mathrm{min}$ for three groups. The SSD (source to surface distance) was a $100 \mathrm{~cm}$ radiation field for $10 \times 10 \mathrm{~cm}$, Three days later, cell viability was measured by MTT assay. At least 3 independent experiments were performed.

\section{Clonogenic assay}

$1 \times 10^{6}$ NK-92 or Hank-1cells were treated with $0,2,4,6$, 8 , and 10 Gy of irradiation, respectively. Twelve days later, the cells were fixed with methanol and stained using 1\% crystal violet in $70 \%$ ethanol. The colonies containing 50 or more cells were counted according to our previous study [17] . The survival curve was derived from a multitarget single-hit model, $y=1-1$-exp.(-D/D0)n [18]. The radiation sensitivity enhancement ratio (SER) was measured according to the multi-target single-hit model. In some experiments, cells were treated with LY294002 
starting $1 \mathrm{~h}$ before radiation for a duration of $24 \mathrm{~h}$. At least 3 independent experiments were performed.

\section{Flow cytometry analysis of cell cycle and apoptosis} $1 \times 10^{6}$ NK-92 or Hank-1 cells of above miR-control and miR-150 group for each well were irradiated with $6 \mathrm{~Gy}$, the cells were collected at $48 \mathrm{~h}$ after radiation. Annexin $\mathrm{V}$ fluorescein isothiocyanate and propidium iodide stains were used to determine the percentage of cells undergoing apoptosis. The apoptosis assay was conducted using the protocol supplied by the manufacturer. Each sample was then subjected to analyses by flow cytometry. At least 3 independent experiments were performed.

\section{Luciferase reporter assay}

The 3' untranslated region (UTR) of AKT2 and AKT3 containing the putative miR-150-binding site was amplified from genomic DNA via PCR using the following primers: wt-AKT2 F, 5'-GCAGTCTGCCCACGCA GAGG-3', and Rv, 5'-TACAG ATGGATAGCTAGTTT A-3', wt-AKT3 F, 5'-GTCTCTTTCATTCTGC TACTT CAC - $3^{\prime}$, and Rv, 5'-GTAAAATGCCCTTTAACCCCC GT-3'. Mutation in $3^{\prime}$-UTR of both genes with miR-150 target binding site deleted was generated with the Quick Change Site-Directed Mutagenesis kit. Both the wild and mutant types of AKT2 and AKT3 genes were cloned into the psiCHECK-2 vector immediately downstream of the Renilla luciferase gene. A luciferase reporter construct containing the miR-150 consensus target sequence served as the positive control, and the psiCHECK-2 vector was used as the internal control. Cells were cotransfected with psiCHECK-2 firefly luciferase reporter (50 ng), psiCHECK-2 Renilla luciferase reporter (10 ng), and miR-150 (50 nM) or miR-control (50 nM) with Lipofectamine 2000 reagent. Cell lysates were prepared using passive lysis buffer for $48 \mathrm{~h}$ upon transfection, and luciferase activity was measured using the DualLuciferase Assay kit and normalized to firefly luciferase activity. Three independent experiments were performed in triplicate.

\section{Western blot (WB)}

Cells were collected in ice-cold PBS $48 \mathrm{~h}$ after transfection and lysed on ice in cold modified RIPA buffer supplemented with protease inhibitors. The concentration of protein was determined by the BCA Protein Assay Kit. Then equal amount of protein were analyzed by SDS-PAGE. Gels were electroblotted onto nitrocellulose membranes, blocked in PBS/dry milk/0.05\% Tween-20, membranes were incubated at $4{ }^{\circ} \mathrm{C}$ over night with first antibodies (Monoclonal rabbit anti-human anti-Akt (ab126433), antip-Akt (ab8805), anti-caspase 3 (ab136812), anti-AKT2 (ab38513), anti-AKT3 (ab152157), anti-mTOR (ab2732) and anti-p-mTOR (ab84400) were all purchased from Cell
Signaling Technology (Beverly, MA, USA), and monoclonal mouse anti-human anti-Alpha-Tubulin (ab7750) and anti-GAPDH (ab8245) were purchased from Kang Chen Bio-tech (shanghai, china), according to the manufacturer's instructions.

\section{In vivo experiment}

Four-week-old male severe combined immunodeficient mice were injected subcutaneously with $2 \times 10^{5}$ Hank-1miR-150 or Hank-1-miR-control cells, separately. When the tumor mass became palpable (about $110 \mathrm{~mm}^{3}$ ), mice in each group were randomly divided into subgroups, including control group, and IR-treated group. Each group contained six mice, and there was no difference in tumor size among the groups. Using this model, we monitored the efficacy of IR (2 Gy/2 days, total $40 \mathrm{~Gy}$ ) in the miR-150 and miR-control groups, respectively. For the control group, dimethyl sulfoxide alone (100\% DMSO, $40 \mathrm{uL}$, intraperitoneal injection every 2 days) was delivered to the mice. Tumor volume (V) was monitored by measuring the length (L) and width (W) of the tumor with calipers and was calculated with the formula $\mathrm{V}=\left(\mathrm{L}^{*} \mathrm{~W}^{2}\right) * 0.5$. All the procedures were in accordance with the guidelines of the Animal Ethics Committee of Southern Medical University.

\section{Statistical analysis}

Statistical analysis was performed with SPSS 16.0 software. All data are presented as the mean $\pm S D$, and analyzed using Student's t-test or analysis of variance (ANOVA) for normal distribution. Comparisons between two/three groups were performed with the Mann-Whitney U-test or the Kruskal-Wallis test for non-normal distribution. A Chi-square test was used to analyze the relationship between miR-150 and the clinical characteristics. $P$-values of $<0.05$ were considered statistically significant.

\section{Results \\ Low levels of miR-150 were frequently found in NKTL tissues and cell lines}

In this study, we detected miR-150 expression levels in NKTL tissues from 36 patients. The expression levels of miR-150 were obviously lower in the tumor tissues than those in normal sCD3 ${ }^{-}$and $\mathrm{CD}^{+} 6^{+} \mathrm{NK}$ cells $(9.10 \pm 0.19$ vs $4.01 \pm 0.22, P<0.05)$. In addition, among five NKTL cell lines examined, four of them (NK-92, SNK-6, HANK-1, SNK-1) had significantly lower expression levels of miR-150 than NK cells obtained from normal peripheral blood. $(P<0.05$, Fig. 1$)$.

\section{miR-150 expression was positively correlated with the treatment response of NKTL patients}

As shown in Table 1, low expression of miR-150 was closely correlated with the viral load of Epstein-Barr virus $(\mathrm{EBV})(\mathrm{EBV} \geqq 50 \mathrm{copy} / \mathrm{ml}$ severse as positive) $(P=$ 

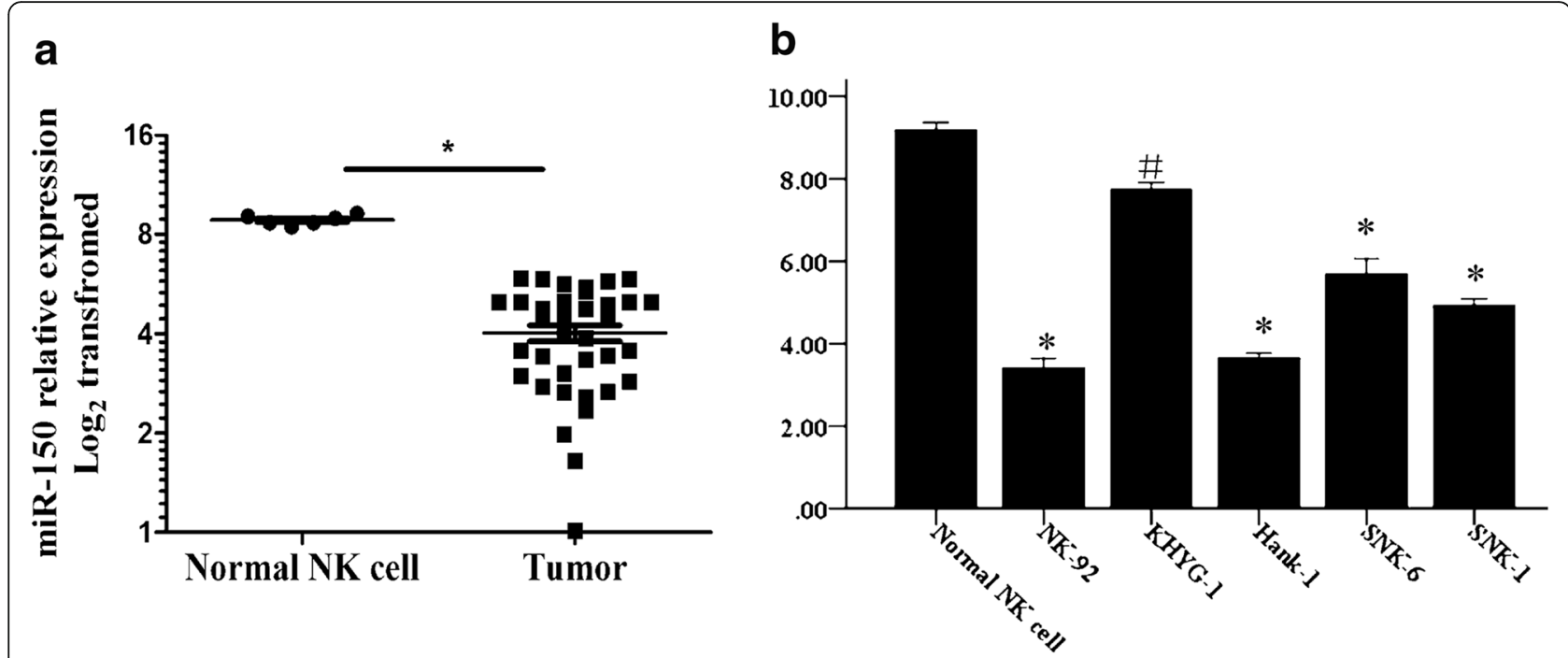

Fig. 1 The expression levels of miR-150 in NKTL tissues and cell lines examined by qRT-PCR. a Expression of miR-150 in 36 samples of NKTL and normal NK cell (Student's t-test); b miR-150 expression levels varied in different NKTL cell lines (Post Hoc test of ANOVA). ${ }^{\#} P<0.05,{ }^{*} P<0.01$

Table 1 Correlation between the expression of miR-150 and clinicopathologic features in NKTL

\begin{tabular}{|c|c|c|c|c|}
\hline & \multirow[b]{2}{*}{ All cases } & \multicolumn{2}{|c|}{ miR-150 expression level } & \multirow[b]{2}{*}{$P$ value } \\
\hline & & Low expression & High expression & \\
\hline Gender & & & & 0.278 \\
\hline female & 16 & $12(75 \%)$ & $4(25 \%)$ & \\
\hline male & 20 & $12(55 \%)$ & $8(40 \%)$ & \\
\hline Age & & & & 0.637 \\
\hline$\geq 40$ years & 15 & $10(66.7 \%)$ & $5(33.3 \%)$ & \\
\hline$<40$ years & 21 & $14(77.8 \%)$ & 7 (22.2\%) & \\
\hline Therapy response & & & & 0.000 \\
\hline$C R$ & 12 & $3(25 \%)$ & $9(75 \%)$ & \\
\hline Non-CR & 24 & $21(87.5 \%)$ & $3(12.5 \%)$ & \\
\hline Clinical stage & & & & 0.133 \\
\hline I & 3 & 1 (33.3\%) & $2(66.7 \%)$ & \\
\hline$\|$ & 7 & $3(42.8 \%)$ & $4(57.2 \%)$ & \\
\hline III & 18 & $15(83.4 \%)$ & $3(16.6 \%)$ & \\
\hline IV & 8 & $5(62.5 \%)$ & $3(37.5 \%)$ & \\
\hline IPI stage & & & & 0.853 \\
\hline 1 & 18 & $13(72.2 \%)$ & $5(27.7 \%)$ & \\
\hline 2 & 14 & 9 (64.2\%) & $5(35.7 \%)$ & \\
\hline 3 & 2 & $1(50 \%)$ & $1(50 \%)$ & \\
\hline 4 & 2 & $1(50 \%)$ & $1(50 \%)$ & \\
\hline EBV & & & & 0.007 \\
\hline normal & 10 & $3(30 \%)$ & $7(70 \%)$ & \\
\hline increase & 26 & $21(80.7 \%)$ & $5(19.2 \%)$ & \\
\hline
\end{tabular}

Mean age; WHO World Health Organization, $C R$ complete response, Non-CR (including $P R$ partial response, NC no change, $P D$ progressive disease)
0.007). No significant association was found between miR-150 expression and other clinico-pathological features, such as the patient's gender, age, clinical stage, and IPI stage $(P>0.05)$. In our enrolled cases, CR, PR, $\mathrm{NC}$, and PD were achieved in 12, 4, 5, and 15 patients at the evaluation time, respectively. Moreover, miR-150 expression was also the factor that showed a positive correlation with treatment response in our enrolled cases, in which low expression of miR-150 was observed more frequently in patients from the non-CR subset than those in the CR subset $(P=0.000)$.

miR-150 enhanced the sensitivity of NKTL cells to IR To explore the underlying mechanism involved in the role of miR-150 in the treatment response of NKTL cells, we induced miR-150 expression upon lentiviral infection in NK-92 and Hank-1 cells, which had the lowest levels of miR-150 among our available NKTL cell lines. Increased expression of miR-150 was confirmed by qRTPCR (Fig. 2a). In the MTT cell proliferation assay, there were no difference between three groups from two cell lines $(P>0.05)$. we tested whether miR-150 increased NKTL cell sensitivity to radiotherapy in vitro. NK-92 and Hank-1 cells were treated with various clinical doses of IR. As shown in Fig. 2c, IR was readily cytotoxic to NK-92 and Hank-1 cells. However, IR resulted in a higher level of cell death in miR-150 group compared to the miR-control and mock group $(P<0.05)$, however, there were no differences between the miR-control and mock group $(P>0.05)$. In addition, we applied colony formation assay by GraphPad Prism 5 software to further confirm that miR-150 increased the sensitivity of NKTL cells to IR in vitro. NKTL cells formed fewer colonies in 


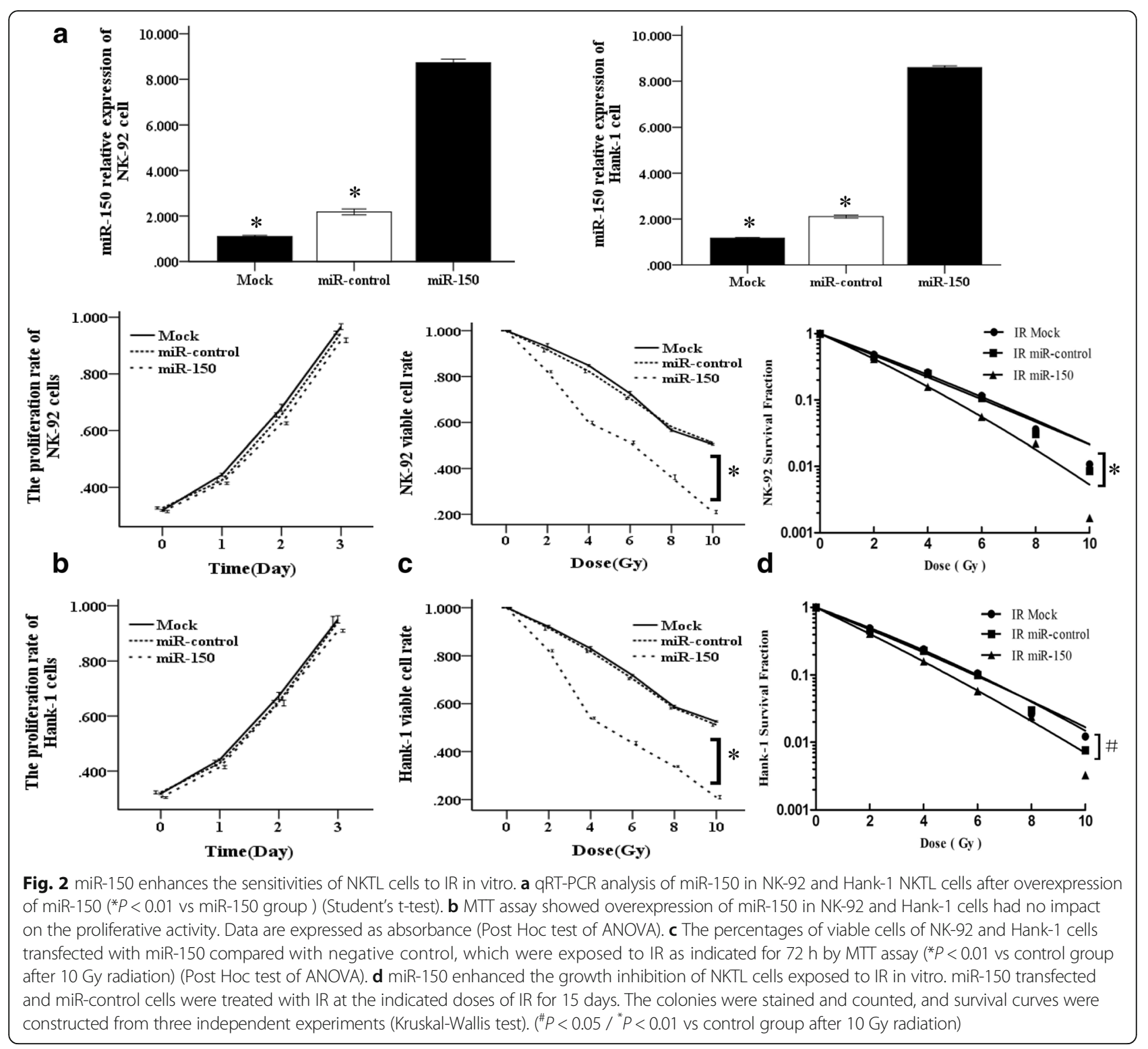

miR-150 groups compared to other group (Fig. 2d), according to the linear quadratic model-fitted cell survival curve. The corresponding biological parameters of the survival curve are shown in Table 2. SERD0, SERDq, and $\mathrm{SERSF}_{2}$ were 1.36, 2.050, and 1.064, respectively. These results provided evidence that miR-150 could increase the sensitivity of NKTL cells to radiotherapy in vitro.

\section{miR-150 promoted IR-induced apoptosis in two cell lines in vitro}

To test whether the miR-150-induced sensitivity to IR was due to activation of apoptosis, we examined the effects of miR-150 on apoptosis in NKTL cell lines using flow cytometry. As shown in Fig. 3a, overexpressions of miR-150 significantly promoted the radiation-induced apoptosis of NK-92 cells $[(39.8 \pm 1.04) \%$ vs $(54.2 \pm 1.12) \%, P<0.05]$ and
Hank-1 cells [(39.8 \pm 1.24$) \%$ vs $(56.2 \pm 1.04) \%, P<0.05]$ for Annexin V/PI analysis when treated with IR (10 Gy). As illustrated in Fig. 3b, the levels of cleaved caspase-3 [(0.16 \pm 0.02 vs $0.40 \pm 0.01, P<0.05)]$ and cleaved PARP $[(0.19 \pm$ 0.03 vs $0.42 \pm 0.01, P<0.05)]$ in Hank-1 cells and the cleaved caspase- $3[(0.10 \pm 0.02$ vs $0.19 \pm 0.01, P<0.05)]$ and cleaved PARP $[(0.14 \pm 0.03$ vs $0.28 \pm 0.01, P<0.05)]$ in NK-92 cells were increased in miR-150 group compared to the miR-control group after treated with IR (10 Gy) by western blot analysis. These data revealed that miR-150 promotes IR-induced apoptosis in NKTL cells.

Both AKT2 and AKT3 are direct targets ofmiR-150 in NKTL cells

We searched for miR-150 targets using three publicly available databases (TargetScan, Pictar and miRBase). 
Table 2 Radiation biology parameters by single-hit multi-target model

\begin{tabular}{lllllll}
\hline Groups & $\mathrm{D} 0$ & $\mathrm{Dq}$ & $\mathrm{SF}_{2}$ & $\mathrm{SER}_{\mathrm{DO}}$ & $\mathrm{SER}_{\mathrm{Dq}}$ & $\mathrm{SERS}_{\mathrm{F} 2}$ \\
\hline Control & 2.7 & 0.37 & 0.474 & - & - & - \\
miR-150 & 1.98 & 0.18 & 0.417 & 1.363 & 2.050 & 1.064 \\
\hline
\end{tabular}

We then used WB analysis to assess expression in various candidate targets, including c-Myb, FOXP1, AKT2, AKT3, BCAP, PRL1, c-Raf, MSK1, MIB1 and E2F3 in NK-92 and Hank-1 cells transduced with miR-150 or empty vector. The PI3K signaling pathway is usually activated in lymphoma, and inactivation of this pathway impairs DNA repair following radiation, then, we found that levels of both AKT2 and AKT3 were reduced in all miR-150 transductants (Fig. 4d). To demonstrate the direct inhibition by miR-150, luciferase reporter containing wild-type AKT2 and AKT3 3'UTR sequences or their mutant-derivates with deletion of putative miR-150- binding sites were cotransfected into both NKTL cell lines with miR-150 (Fig. 4a). As shown in Fig. 4b, miR150 suppressed luciferase activities depending on the presence of miR-150 binding in the wild-type AKT2 and AKT3 3'UTR. Upon 3'UTR mutation of AKT2 and AKT3, miR-150 can not bind to its target site, resulting in increased luciferase activity. The ratio of wild-type AKT2 and AKT3 were decreased upon miR-150 overexpression in Hank-1 and NK-92 cell lines (Fig. 4c). Morever, overexpression of miR-150 significantly suppressed the protein expression of AKT2 and AKT3 in NK-92 and Hank-1 cells using WB analysis (Fig. 4d), All these results together suggested that AKT2 and AKT3 direct targets of miR-150 in NKTL cells.

miR-150 induces NKTL cells radiosensitivity by inhibiting the PI3K/AKT/mTOR pathway

mTOR is the downstream molecular of AKT signaling pathways. We found that overexpression of miR-150

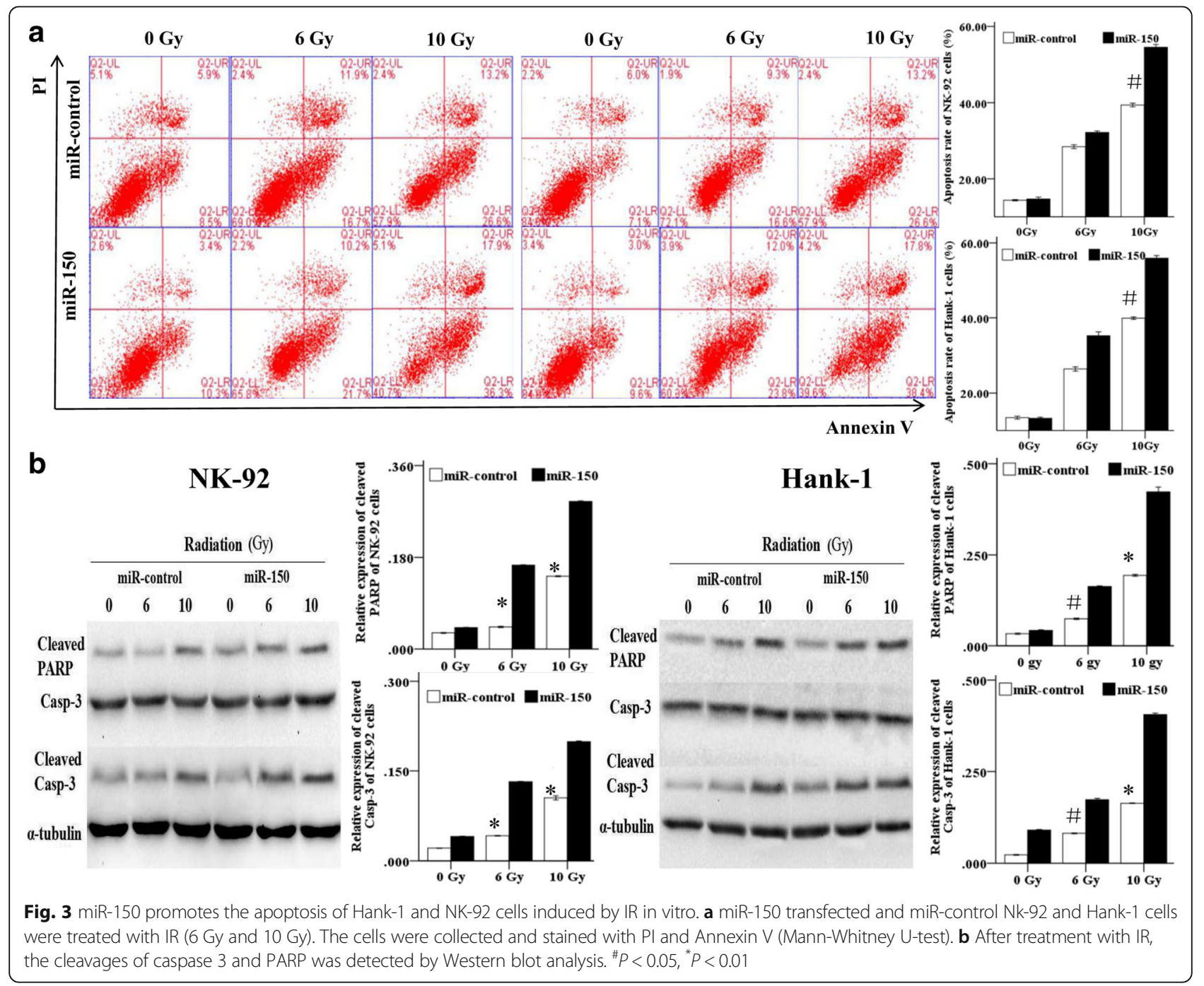



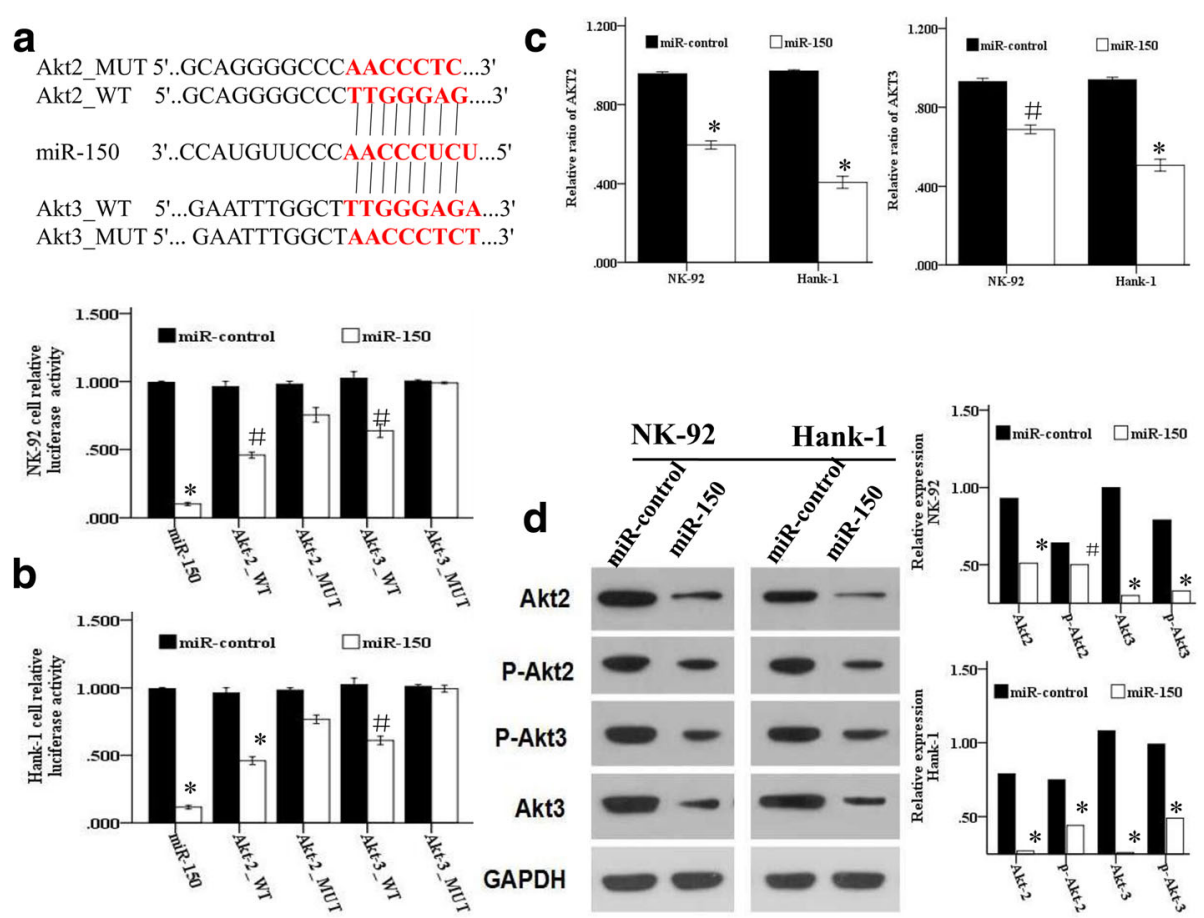

Fig. 4 AKT2 and AKT3 are direct targets of miR-150 in NKTL. a Schematic representation of AKT2 and AKT3 UTR showing putative miR-150 target sites; $\mathbf{b}$ Relative luciferase activity were performed to detect the expression of wild-type/mutant AKT2 and AKT3 upon transfection with miR-150 or miR-control (normalized to firefly luciferase activity); c Relative ratio of AKT2 and AKT3 reporter constructs in Hank-1 and NK-92 cell lines, co-transfected with miR-150 or miR-control is shown; $\mathbf{d}$ Western blot analysis showed the expression levels of total AKT, p-AKT, AKT2 and AKT3 proteins in NKTL cells treated with miR-150 and miR-control. (Student's t-test). ${ }^{\#} P<0.05,{ }^{*} P<0.01$

reduced the expression of AKT meanwhile decreased the expression of p-mTOR by WB analysis. Moreover, we found that exposure to the tyrosinekinase inhibitor LY294002 activating the effect of miR-150 resulted in decrease of $\mathrm{p}-\mathrm{AKT} / \mathrm{p}-\mathrm{mTOR}$ expresssion and formed colonies after different dose of radiation. There were statistical difference between the four groups in NK92 cell $\left(X^{2}=11.26 P=0.021\right)$ and Hank-1 cell $\left(X^{2}=\right.$ $10.38 P=0.016)$ after 10 Gy radiation, and the total clone formation rate was minimum in the miR-150 + LY294002 group according to mean rank (Fig. 5a and b). Those function experiment confirmed that AKT pathway was the mechanism by which miR-150 sensitizes cells toward radiotherapy.

miR-150 enhanced the sensitivity of Hank-1 cells to IR in vivo

Freshly isolated Hank-1-miR-control and Hank-1-miR150 cells were transplanted into NODSCID-12 IL-2 RG -/ - (NSI) mice(purchased from Guangzhou Institutes of Biomedicine and Health (GIBH)) to establish P1 NKTL mice according to protocols [19]. We next examined the anti-tumor effect of miR-150 on NKTL xenografts treated with IR. The tumors were irradiated with 2 Gy every 2 day (total dose $=40$ Gy) after tumor mass became palpable, followed by detection of NKTL xenograft growth (Fig. 6). It can be seen that the tumor volume was significantly decreased in the radiotherapy group (from day 10 to $30, P<0.01$ ) and combined therapy group (from day 10 to $30, P<0.01$ ) compared with control group. The tumor volume of control group showed no significant change compared with miR-150 group (from day 5 to $20, P>0.05$ ). The average tumor volume reached $1587.96 \pm 83.56 \mathrm{~mm}^{3}$ in control group on day 30 , while only $449.58 \pm 29.20 \mathrm{~mm}^{3}$ in combined therapy group, $1448.02 \pm 96.99 \mathrm{~mm} 3$ in miR-150 therapy group, and $1012.95 \pm 78.77 \mathrm{~mm}^{3}$ in radiotherapy group. In addition, the tumor volume was significantly decreased in mice of combined therapy group compared with miR150 therapy (from day 10 to $30, P<0.01$ ) or radiotherapy group (from day 15 to $30, P<0.01$ ).

\section{Discussion}

In the current study, we found a significant decrease of miR-150 in NKTL biopsies compared with that in normal NK cells. This observation is consistent with the results of several previous reports $[15,20]$. Our further correlation analysis demonstrated that decreased levels of miR-150 were closely associated with certain aggressive features of NKTL, such as EBV viral load, and several studies have confirmed the association of an aggressive lymphoma to EBV [21-23]. Chen et al. found 


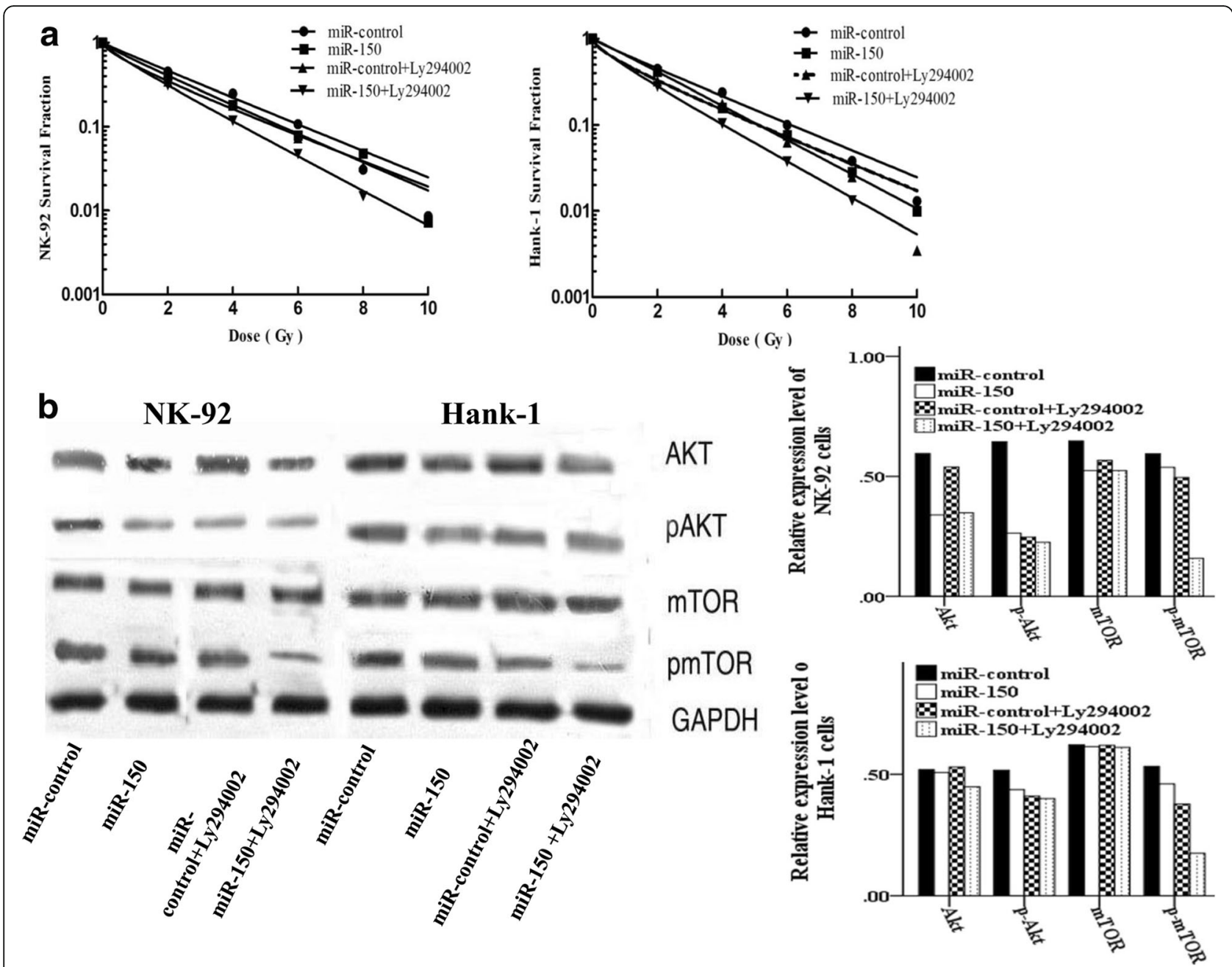

Fig. 5 The PI3K tyrosine kinase inhibitor LY294002 synergistic effect of miR-150. a The NKTL cells lines NK-92 and Hank-1 were transfected with miR-150 or miR-control. The cells were then treated with or without LY294002 for $1 \mathrm{~h}$ before exposure to different doses radiation. Survival fractions were calculated and analyzed (Kruskal-Wallis test); b Western blot analysis of p-mTOR and p-Akt levels in NK-92 and Hank-1 cells transfected with miR-150 or miR-control, then treated with LY294002 for $1 \mathrm{~h}$, and finally exposed to a 6 Gy dose of radiation for another $24 \mathrm{~h} .{ }^{\#} P<0.05,{ }^{*} P<0.01$

that overexpression of miR-150 could induce EBVpositive Burkitt lymphoma to differentiate into a more mature stage [21]. Moreover, we found that low expression of miR-150 in NKTL was correlated closely with poor response to treatment. Thus, our reports suggested that the examination of miR-150 expression could be used as an effective additional tool to predict the therapeutic response of NKTL.

Based on the above findings, we hypothesize whether reintroduction of miR-150 could rescue the therapeutic sensitivity in NKTL cells. Until now, several microRNAs have been reported to participate in regulating therapeutic efficacy in cancer therapy [24-26]. Our present research demonstrated, for the first time, that miR-150 could substantially sensitize NKTL cells to radiation either in vitro and in vivo. The efficacy of radiation is generally believed to depend on their ability to trigger apoptosis in tumor cells. Thus, we further examined the effect of miR-150 on the IR-induced apoptosis in NK-92 and Hank-1 cells using flow cytometry and WB. We found that miR-150 could enhance apoptotic cell induced by IR. These findings strongly suggest that overexpression of miR-150 into NKTL cells sensitize NKTL cells to IR-induced apoptosis.

EBV plays an important role in the lymphomagenesis of NKTL [27]. Latent membrane protein 1 (LMP1) is a major oncoprotein that is encoded by one of the EBV genes [28]. This protein contributes to the activation of the AKT pathway in EBV-infected lymphomas, which, in turn, affects cell survival, apoptosis, proliferation, and genomic instability via its downstream target proteins to cause cancer [29]. Moreover, Huang et al. confirmed that AKT signaling pathways may contribute to the angiogenesis, proliferation, and survival of NKTL [26]. It has 


\section{a}

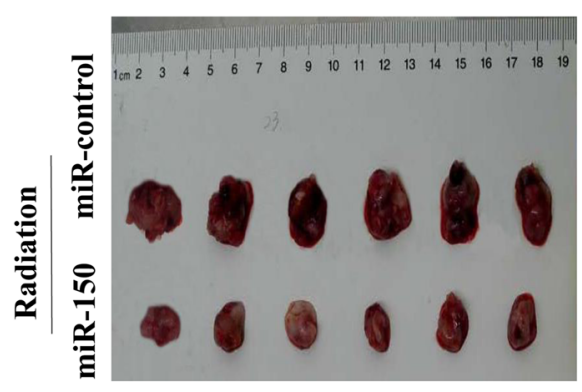

b

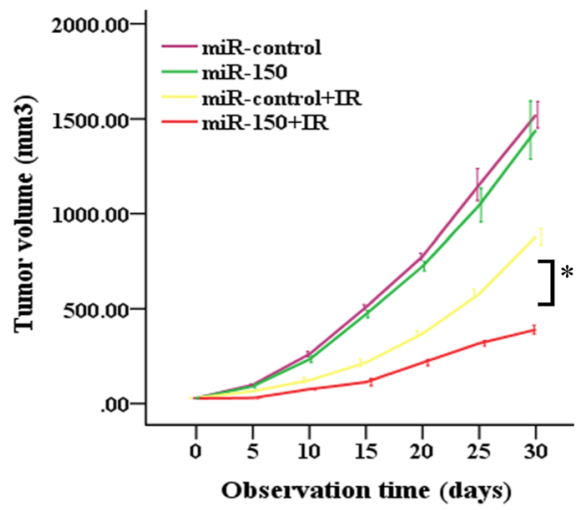

Fig. 6 miR-150 enhances the sensitivities of Hank-1 cells to IR in vivo. a Representative images of xenograft tumours in NODSCID-12 IL2RG-/(NSI) mice induced by Hank-1-miR-150 or Hank-1-miR-control cells. The tumor volumes after treatment with IR within 30 days of two groups. $\mathbf{b}$ The tumor volumes were measured and recorded every 5 days (the groups related to IR). The tumor growth curves were created from 6 mice in each group (Post Hoc test of ANOVA). ${ }^{*} P<0.01$

also been demonstrated that the activation of the PI3K/ AKT pathway can result in resistance to radiotherapy and chemotherapy [30,31], and inhibition of this pathway can increase the sensitivity of cancer cells to therapy $[32,33]$. Several studies have confirmed that the miR-150 regulation mechanism was related to AKT activation [15, 34-36]. Luciferase assays confirmed that the mRNA and protein levels of both wild-type AKT2 and AKT3 could be consistently decreased in NKTL cells through transfection with miR-150 (Fig. 4), moreover, overexpression of miR150 inhabited the PI3K/AKT/mTOR signaling molecules, and that the PI3K tyrosine kinase inhibitor LY294002 enhanced the effects of miR-150, indicating that the PI3K/ $\mathrm{AKT} / \mathrm{mTOR}$ signaling pathway is involved in miR-150 mediated radiosensitivity.

\section{Conclusions}

In summary, our report describes the expression pattern of miR-150 in human NKTL, and low expression of miR-150 may be important in the acquisition of poor therapeutic response of the tumor. Furthermore, functional studies of miR-150 suggest a critical role of miR150 in enhancing the sensitivities of NKTL to radiotherapy by suppressing PI3K/AKT/mTOR signaling.

\section{Additional file}

Additional file 1: Supplementary Materials and Methods. (DOCX $49 \mathrm{~kb})$

\section{Acknowledgments}

The authors thank Qiang Xie for technical assistance with the irradiation protocol and Yu Fa Li for pathological assistance. We would like to thank professor YuHua Li because she made great contribution on analysis and interpretation of data in the period of a major revision of the manuscript. This work was supported by the National Natural Science Foundation of China (No. 81400156).

\section{Authors' contributions}

SJW designed the work, acquisition, analysis and interpretation of data, wrote the manuscript; BYW, JC analyzed data and reviewed critically the manuscript for important intellectual content; KYG, YJW designed the work and acquisition of data. All authors read and approved the final manuscript.

\section{Competing interests}

The authors declare no conflicts of interest to disclosure.

\section{Publisher's Note}

Springer Nature remains neutral with regard to jurisdictional claims in published maps and institutional affiliations.

\section{Author details}

'Department of Hematology, Southern Medical University, Zhujiang Hospital, 253\# industry road, Guangzhou, Guangdong 510282, China. ${ }^{2}$ Department of Radiotherapy, Southern Medical University, Zhujiang Hospital, 253\# industry road, Guangzhou, Guangdong 510282, China. ${ }^{3}$ Department of Laboratory Animal Center, Southern Medical University, 1838 Guangzhou North Road, Guangzhou, Guangdong 510515, China.

Received: 20 June 2017 Accepted: 16 November 2017

Published online: 31 January 2018

\section{References}

1. Au WY, Weisenburger DD, Intragumtornchai T, et al. Clinical differences between nasal and extranasal natural killer/T-cell lymphoma: a study of 136 cases from the international peripheral T-cell lymphoma project. Blood. 2009:113:3931-7.

2. Ma X, Guo Y, Pang Z, et al. A randomized phase II study of CEOP with or without semustine as induction chemotherapy in patients with stage IE/IIE extranodal NKT-cell lymphoma, nasal type in the upper aerodigestive tract. Radiother Oncol. 2009;93:492-7.

3. Nowarski R, Wilner OI, Cheshin O, et al. APOBEC3G enhances lymphoma cell radioresistance by promoting cytidine deaminase-dependent DNA repair. Blood. 2012;120:366-75.

4. Li Q, Xu X, Zhong W, et al. LL-17 induces radiation resistance of B lymphoma cells by suppressing p53 expression and thereby inhibiting irradiation-triggered apoptosis. CELL MOL IMMUNOL. 2015;12:366-72.

5. Tse E, Kwong YL. The diagnosis and management of NK/T-cell lymphomas. J Hematol Oncol. 2017;10:85.

6. Bartel DP. MicroRNAs: target recognition and regulatory functions. Cell. 2009;136:215-33.

7. Kozloski GA, Lossos IS. LymphomiRs: microRNAs with regulatory roles in lymphomas. Curr Opin Hematol. 2015;22:362-8. 
8. Jiang P, Rao EY, Meng N, Zhao Y, Wang JJ. MicroRNA-17-92 significantly enhances radioresistance in human mantle cell lymphoma cells. Radiat Oncol. 2010;5:100.

9. Zhang P, Wang L, Rodriguez-Aguayo C, et al. miR-205 acts as a tumour radiosensitizer by targeting ZEB1 and Ubc13. Nat Commun. 2014;5:5671.

10. Vasilatou D, Papageorgiou S, Pappa V, Papageorgiou E, Dervenoulas J. The role of microRNAs in normal and malignant hematopoiesis. Eur J Haematol. 2010;84:1-16

11. Bezman NA, Chakraborty $T$, Bender T, Lanier LL. miR-150 regulates the development of NK and iNKT cells. J Exp Med. 2011;208:2717-31.

12. Tano N, Kim HW, Ashraf M. microRNA-150 regulates mobilization and migration of bone marrow-derived mononuclear cells by targeting Cxcr4. PLoS One. 2011:6:e23114.

13. He $Y$, Jiang $X$, Chen J. The role of miR-150 in normal and malignant hematopoiesis. Oncogene. 2014;33:3887-93.

14. Wang F, Ren X, Zhang X. Role of microRNA-150 in solid tumors. Oncol Lett. 2015;10:11-6.

15. Watanabe A, Tagawa H, Yamashita J, et al. The role of microRNA-150 as a tumor suppressor in malignant lymphoma. Leukemia. 2011;25:1324-34.

16. Yang H, Fu G, Liu J, et al. Clinical analysis of 42 cases of EBV-positive mature T/NK-cell neoplasms. EXP THER MED. 2017;14:567-74.

17. Zhang S, Hao J, Xie F, et al. Downregulation of miR-132 by promoter methylation contributes to pancreatic cancer development. Carcinogenesis. 2011;32:1183-9.

18. Ke G, Liang L, Yang JM, et al. MiR-181a confers resistance of cervical cancer to radiation therapy through targeting the pro-apoptotic PRKCD gene. Oncogene. 2013:32:3019-27.

19. Ye W, Jiang Z, Li GX, et al. Quantitative evaluation of the immunodeficiency of a mouse strain by tumor engraftments. J Hematol Oncol. 2015;8:59.

20. Machova PK, Lopotova T, Klamova $\mathrm{H}$, et al. Expression patterns of microRNAs associated with CML phases and their disease related targets. Mol Cancer. 2011;10:41.

21. Chen S, Wang Z, Dai $X$, et al. Re-expression of microRNA-150 induces EBVpositive Burkitt lymphoma differentiation by modulating c-Myb in vitro. Cancer Sci. 2013;104:826-34.

22. Sousa J, Cabezuelo L, Almeida S, et al. Aggressive NK/T cell leukemia/ lymphoma associated with EBV. Acta Medica Port. 2011;24(Suppl 3):649-52.

23. Jung KS, Cho SH, Kim SJ, Ko YH, Kim WS. Clinical features and treatment outcome of Epstein-Barr virus-positive nodal T-cell lymphoma. Int J Hematol. 2016;104:591-5.

24. Zhang JX, Qian D, Wang FW, et al. MicroRNA-29c enhances the sensitivities of human nasopharyngeal carcinoma to cisplatin-based chemotherapy and radiotherapy. Cancer Lett. 2013;329:91-8.

25. Gong P, Zhang T, He D, Hsieh JT. MicroRNA-145 modulates tumor sensitivity to radiation in prostate cancer. Radiat Res. 2015;184:630-8.

26. Huang $Y$, de Reynies A, de Leval $L$, et al. Gene expression profiling identifies emerging oncogenic pathways operating in extranodal NK/T-cell lymphoma, nasal type. Blood. 2010;115:1226-37.

27. Zhang T, Fu Q, Gao D, et al. EBV associated lymphomas in 2008 WHO classification. Pathol Res Pract. 2014;210:69-73.

28. Halabi MA, Jaccard A, Moulinas R, et al. Clonal deleted latent membrane protein 1 variants of Epstein-Barr virus are predominant in European extranodal NK/T lymphomas and disappear during successful treatment. Int J Cancer. 2016;139:793-802

29. Yang CF, Yang GD, Huang TJ, et al. EB-virus latent membrane protein 1 potentiates the stemness of nasopharyngeal carcinoma via preferential activation of PI3K AKT pathway by a positive feedback loop. Oncogene. 2016;35:3419-31

30. Martini M, De Santis MC, Braccini L, Gulluni F, Hirsch E. PI3KVAKT signaling pathway and cancer: an updated review. Ann Med. 2014;46:372-83.

31. Cani A, Simioni C, Martelli AM, et al. Triple Akt inhibition as a new therapeutic strategy in T-cell acute lymphoblastic leukemia. Oncotarget. 2015;6:6597-610.

32. Westin JR. Status of PI3K/Akt/mTOR pathway inhibitors in lymphoma. Clin Lymphoma Myeloma Leuk. 2014;14:335-42.

33. Sun $L$, Zhao $Y$, Shi $H$, Ma C, Wei L. LMP-1 induces survivin expression to inhibit cell apoptosis through the NF-kappaB and PI3K/Akt signaling pathways in nasal NK/T-cell lymphoma. Oncol Rep. 2015;33:2253-60.

34. Wuerkenbieke D, Wang J, Li Y, Ma C. miRNA-150 downregulation promotes pertuzumab resistance in ovarian cancer cells via AKT activation. Arch Gynecol Obstet. 2015;292:1109-16.
35. Sang W, Sun C, Zhang C, et al. MicroRNA-150 negatively regulates the function of CD4(+) T cells through AKT3/Bim signaling pathway. Cell Immunol. 2016;306-307:35-40

36. Chen W, Han C, Zhang J, et al. miR-150 deficiency protects against FASinduced acute liver injury in mice through regulation of AKT. PLOS One. 2015;10:e132734.

\section{Submit your next manuscript to BioMed Central and we will help you at every step:}

- We accept pre-submission inquiries

- Our selector tool helps you to find the most relevant journal

- We provide round the clock customer support

- Convenient online submission

- Thorough peer review

- Inclusion in PubMed and all major indexing services

- Maximum visibility for your research

Submit your manuscript at www.biomedcentral.com/submit

) Biomed Central 